\section{Fenofibrate reduces osteonecrosis without affecting antileukemic efficacy in dexamethasone-treated mice}

\author{
Emily R. Finch, ${ }^{1}$ Monique A. Payton, ${ }^{1}$ David A. Jenkins, ${ }^{1}$ Xiangjun Cai, ${ }^{1}$ Lie $\mathrm{Li},{ }^{1}$ \\ Seth E. Karol, ${ }^{2}$ Mary V. Relling ${ }^{1}$ and Laura J. Janke ${ }^{3}$ \\ ${ }^{1}$ Department of Pharmaceutical Sciences, St. Jude Children's Research Hospital; \\ ${ }^{2}$ Department of Oncology, St. Jude Children's Research Hospital and 'Department of \\ Pathology, Division of Comparative Pathology, St. Jude Children's Research Hospital, \\ Memphis, TN, USA
}

\section{ABSTRACT}

$\mathrm{R}$ ecent clinical trials in children with acute lymphoblastic leukemia (ALL) indicate that severe hypertriglyceridemia (>1000 mg/dL) dur- ing therapy is associated with an increased frequency of symptomatic osteonecrosis. Interventions to lower triglycerides have been considered, but there have been no preclinical studies investigating the impact of lowering triglycerides on osteonecrosis risk, nor whether such interventions interfere with the antileukemic efficacy of ALL treatment. We utilized our clinically relevant mouse model of dexamethasoneinduced osteonecrosis to determine whether fenofibrate decreased osteonecrosis. To test whether fenofibrate affected the antileukemic efficacy of dexamethasone, we utilized a BCR-ABL ${ }^{+}$model of ALL. Serum triglycerides were reduced by fenofibrate throughout the period of treatment, with the most pronounced, 4.5 -fold, decrease at week $3\left(P<1 \times 10^{-6}\right)$. Both frequency ( $33 \%$ vs. $74 \%, P=0.006$ ) and severity (median necrosis score of 0 vs. $75 ; P=6 \times 10^{-5}$ ) of osteonecrosis were reduced with fenofibrate. Fenofibrate had no impact on $B C R-\mathrm{ABL}^{+} \mathrm{ALL}$ survival $(P=0.65)$ nor on the antileukemic properties of dexamethasone $(P=0.49)$. These data suggest that lowering triglycerides with fenofibrate reduces dexamethasone-induced osteonecrosis while maintaining antileukemic efficacy, and thus may be considered for clinical trials.

\section{Introduction}

Hypertriglyceridemia occurs in $4-19 \%$ of children treated with glucocorticoids and/or asparaginase during therapy for acute lymphoblastic leukemia (ALL) ${ }^{1-7}$ Although hypertriglyceridemia has been thought to be transient and relatively benign, ${ }^{1,2}$ recent studies suggest that patients with severe hypertriglyceridemia (>1000 mg/dL) during ALL therapy may be at increased risk of long-term complications, including symptomatic osteonecrosis., ${ }^{3,7,8}$

Recent recommendations for pharmaceutical management of severe hypertriglyceridemia include the use of fibrates, specifically fenofibrate. ${ }^{9}$ In addition to being associated with fewer drug-drug interactions than statins, ${ }^{10-12}$ fenofibrate specifically reduces serum triglycerides by as much as $50 \%$, with minimal adverse reactions, ${ }^{13-15}$ whereas the main effect of statins is the decrease of serum low-density lipoprotein cholesterol. ${ }^{9,16,17}$ The active metabolite of fenofibrate, fenofibric acid, targets peroxisome proliferator activated receptor- $\alpha$, leading to activation of lipoprotein lipase which increases lipolysis and elimination of triglyceride-rich particles, ${ }^{18}$ resulting in reduction of circulating serum triglycerides. ${ }^{19,20}$ Therefore, it has been suggested that fenofibrate could be useful in the management of acute hypertriglyceridemia, such as that observed during ALL therapy. However, there is no evidence that lowering triglyceride levels with fenofibrate can reduce risk of osteonecrosis. Moreover, with very effective ALL therapy, caution must be exercised before adding any new agents to treatment regimens, to avoid untoward drug interactions.

In these proof-of-principle experiments, we tested the hypothesis that lowering serum triglyceride levels with fenofibrate in dexamethasone-treated mice would
Ferrata Storti Foundation
Haematologica 2021

Volume 106(8):2095-2101

\section{Correspondence:}

LAURA JANKE

laura.janke@stjude.org

Received: March 18, 2020.

Accepted: July 6, 2020.

Pre-published: July 16, 2020.

https://doi.org/10.3324/haematol.2020.252767

(C)2021 Ferrata Storti Foundation

Material published in Haematologica is covered by copyright. All rights are reserved to the Ferrata Storti Foundation. Use of published material is allowed under the following terms and conditions:

https://creativecommons.org/licenses/by-nc/4.0/legalcode. Copies of published material are allowed for personal or internal use. Sharing published material for non-commercial purposes is subject to the following conditions:

https://creativecommons.org/licenses/by-nc/4.0/legalcode, sect. 3. Reproducing and sharing published material for commercial purposes is not allowed without permission in writing from the publisher. 
result in decreased osteonecrosis and would not interfere with the efficacy of antileukemic therapy.

\section{Methods}

\section{Treatment}

Experiments were approved by the Institutional Animal Care and Use Committee of St. Jude Children's Research Hospital (SJCRH; Memphis, TN, USA): protocol numbers 423-100428 and 465-100549.

All mice received prophylactic antibiotics in drinking water (herein referred to as "base-water"): continuous tetracycline (1 g/L; Sigma-Aldrich, St. Louis, MO, USA) and intermittent sulfamethoxazole/trimethoprim oral suspension (600/120 mg/L for 3.5 days/week; Aurobindo Pharma, USA, Inc., Dayton, NJ, USA). Mice were randomized to receive continuous dexamethasone $(0.4 \mathrm{mg} / \mathrm{mL}$ sodium phosphate solution; Fresenius Kabi, Lake Zurich, IL, USA) in drinking water, herein referred to as "dexamethasone water" ( $3 \mathrm{mg} / \mathrm{L}$ in the osteonecrosis model and $4 \mathrm{mg} / \mathrm{L}$ in the BCR-ABL ${ }^{+}$ALL model). ${ }^{21-23}$

The folic acid-deficient diet $(0.2 \mathrm{ppm}$ folic acid; TestDiet, Richmond, IN, USA) was the "base-diet". ${ }^{21}$ Fenofibrate (Sigma Aldrich, St. Louis, MO, USA) was added to the base-diet for a final concentration of $0.2 \%$ fenofibrate $(\mathrm{w} / \mathrm{w})$ to make the "fenofibrate-supplemented diet" (TestDiet, Richmond, IN, USA). This dose had been used in prior experimental rodent models. ${ }^{24-26}$

Serum triglycerides were measured with an ABX Penta 400 instrument (Horiba, Montpellier, France) after the animals had fasted for 12 to $16 \mathrm{~h}$ (food was withheld; mice had free access to water).
Mice were randomized to one of four treatment groups: control (base-water and base-diet); fenofibrate-only (base-water and fenofibrate-supplemented diet); dexamethasone-only (dexamethasone-water and base-diet); or dexamethasone + fenofibrate (dexamethasone-water and fenofibrate-supplemented diet).

\section{Osteonecrosis model}

The protocol was modified from that previously described. ${ }^{21,22,27}$ ${ }^{29}$ At postnatal days 26 to 28 , male Balb/cJ mice (bred in-house at SJCRH) were randomized by body weight $(13.5 \mathrm{~g}, 95 \%$ confidence interval $[95 \% \mathrm{CI}]: 13.0-14.7)$ to 6 weeks of treatment. Fasting serum triglycerides were measured after 1, 3, and 6 weeks of treatment. At the end of treatment, white adipose tissue; and plasma dexamethasone and fenofibric acid levels were measured. Osteonecrosis and epiphyseal arteriopathy were evaluated and scored in both distal femora, by a board-certified veterinary pathologist (LJJ), as described previously ${ }^{21,22,27-29}$ Details are provided in the Online Supplementary Methods.

\section{BCR-ABL ${ }^{+}$acute lymphoblastic leukemia model}

The BCR-ABL (p185', Arf ${ }^{\prime}$ ) luciferase-positive cell line (BCR$\left.\mathrm{ABL}^{+}\right)$was generated. ${ }^{23,30-32}$ Female 8-week-old matched syngeneic mice (C57Bl/6J, Arf-wildtype; Jackson Laboratory, Bar Harbor, $\mathrm{ME}, \mathrm{USA}$ ) received intravenous injections of $2,000 \mathrm{BCR}-\mathrm{ABL}^{+}$ cells. Bioluminescent imaging was performed weekly, ${ }^{23}$ to monitor leukemic burden. At day 3, mice were stratified by luminescent signal and body weight to treatment ( $n=10 /$ treatment group). Fasting serum triglycerides were measured at day 24 . Treatment ended at day 28: mice were maintained on basediet/base-water until a humane endpoint or the end of study at day 63. Details are given in the Online Supplementary Methods.

A

\begin{tabular}{|c|c|}
\hline $\begin{array}{l}\text { Dex } \\
\text { (Water) }\end{array}$ & $\begin{array}{c}\text { Fenofibrate } \\
\text { (Diet) }\end{array}$ \\
\hline $0 \mathrm{mg} / \mathrm{L}$ & $0.0 \% \mathrm{w} / \mathrm{w}$ \\
\hline $0 \mathrm{mg} / \mathrm{L}$ & $0.2 \% \mathrm{w} / \mathrm{w}$ \\
\hline $3 \mathrm{mg} / \mathrm{L}$ & $0.0 \% \mathrm{w} / \mathrm{w}$ \\
\hline $3 \mathrm{mg} / \mathrm{L}$ & $0.2 \% \mathrm{w} / \mathrm{w}$ \\
\hline
\end{tabular}

B

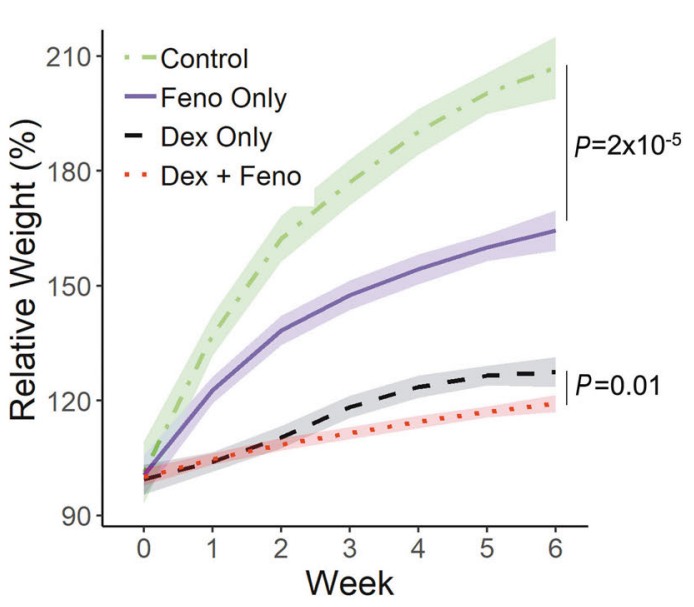

C

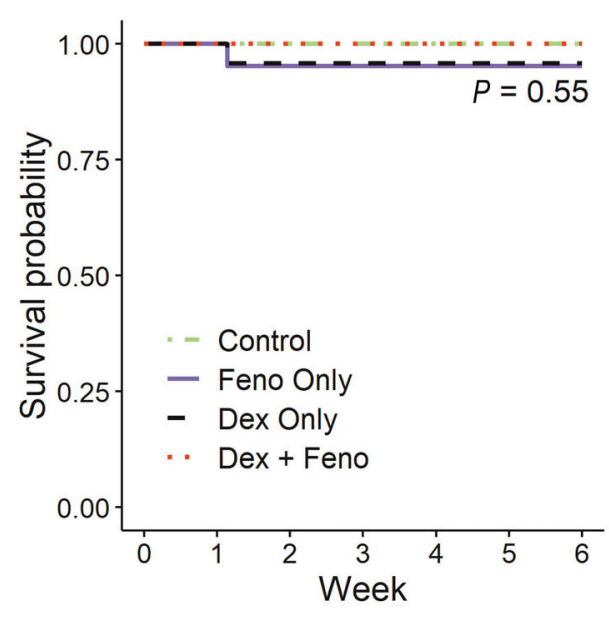

Figure 1. Experimental design, body weight, and survival in dexamethasone-induced osteonecrosis. (A) Experimental design: male Balb/cJ mice were placed on 6 weeks of continuous treatment with dexamethasone, fenofibrate, or both. (B) Body weight relative to week 0 is shown as a median with $95 \%$ confidence interval $(95 \% \mathrm{Cl})$. Control mice and mice treated with fenofibrate (Feno) only weighed more than those treated with dexamethasone (Dex) only or Dex + Feno from weeks 1-6 of treatment $\left(P<1 \times 10^{-6}\right)$. (C) Survival did not differ by treatment group. The survival rate was $100 \%$ in the control group (12/12) and the group treated with Dex + Feno (33/33), whereas it was $98 \%$ in both the Dex Only $(23 / 24)$ and Feno Only $(20 / 21)$ groups $(P=0.55)$. 


\section{Statistical analysis}

The frequency of osteonecrosis/arteriopathy was compared using the $\chi^{2}$ test. Log-rank testing was used for survival comparisons. For continuous variables, the two-tailed Mann-Whitney test was used (two groups); the Kruskal-Wallis test with Dunn correction was used for multiple comparisons (three or more groups). All continuous statistics are expressed as the median with $95 \%$ confidence interval. The R program (version 3.5.1) ${ }^{33}$ or GraphPad Prism ${ }^{34}$ was used for the statistical analyses and visual representation. $P$ values $<0.05$ were considered statistically significant for all analyses.

\section{Results}

\section{Fenofibrate in experimental osteonecrosis}

Consistent with previous observations, ${ }^{21,22,28,29}$ mice that received dexamethasone (i.e., animals in the dexamethasone-only and dexamethasone + fenofibrate treatment groups) gained less weight over time compared to those not treated with dexamethasone (control and fenofibrate- only treatment groups) (Figure 1B). Fenofibrate supplementation suppressed weight gain over the course of treatment. By week 6 of treatment, control mice had gained $50 \%$ more weight than mice treated with fenofibrate only $\left(P=2 \times 10^{-5}\right)$ and mice treated with dexamethasone only had gained $10 \%$ more weight than those treated with dexamethasone + fenofibrate $(P=0.01)$ (Figure $1 \mathrm{~B})$. There was no difference in survival between groups $(P=0.55)$ (Figure 1C).

In mice treated with dexamethasone, fenofibrate significantly reduced serum triglyceride levels as early as week 1, from $130.1 \mathrm{mg} / \mathrm{dL}$ (95\% CI: $85.2-201.1$ ) to $37.4 \mathrm{mg} / \mathrm{dL}$ (95\% CI: $17.1-60.3)$, a 3.5-fold decrease $(P=0.0004)$. Fenofibrate supplementation continued to suppress dexamethasone-induced hypertriglyceridemia at week 3 (4.5fold decrease, $\left.P<1 \times 10^{-6}\right)$ through to the end of treatment at week 6 (3.5-fold decrease, $\left.P=5 \times 10^{-6}\right)$. There was no difference in triglyceride levels at weeks 1 and 3 between mice treated with fenofibrate only and those treated with

A

Control

- Fenofibrate Only

$\triangle$ DEX Only

$\checkmark$ DEX + Fenofibrate

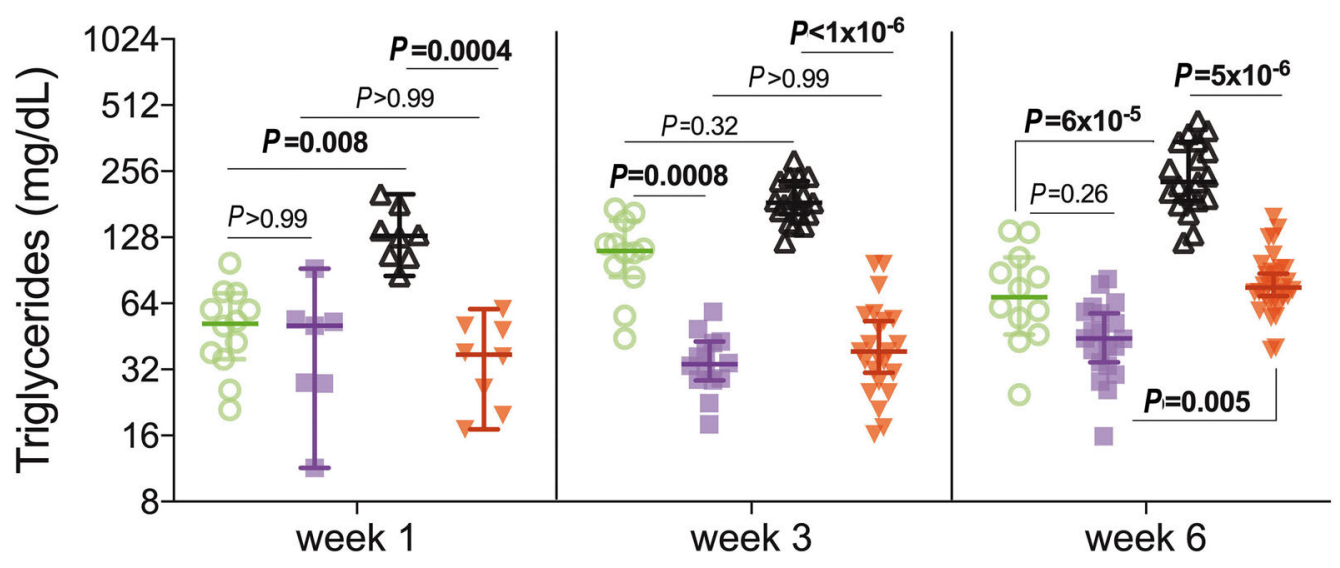

B

Perigonadal

Inguinal

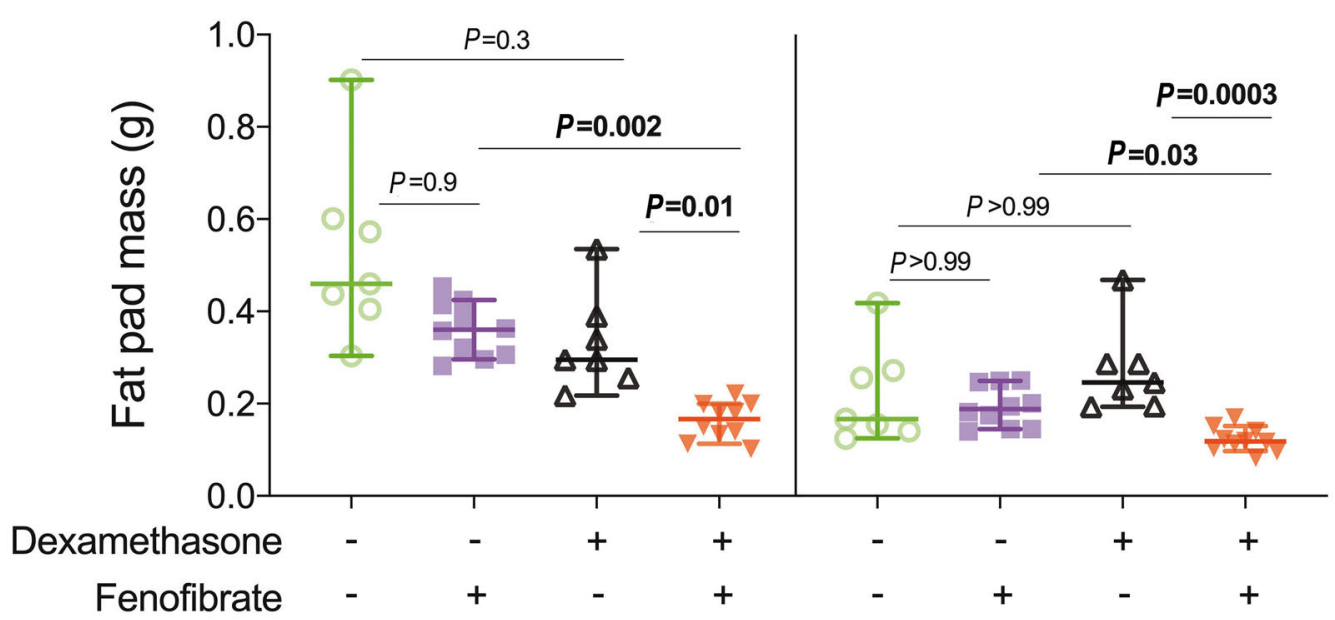

Figure 2. Fenofibrate reduces serum lipids and fat depots in dexamethasone-treated mice. (A) Median (with 95\% confidence interval) fasting serum triglyceride levels at weeks 1, 3, and 6 of treatment. Week 1, n=8-12/group; week 3, n=12-27/group; week 6, n=12-31/group. (B) Perigonadal and inguinal fat pads at week 6; $\mathrm{n}=8-10$ /group. $P$-values shown between relevant groups: control versus fenofibrate only; control versus dexamethasone (DEX) only; fenofibrate only versus DEX + fenofibrate; DEX only versus DEX + fenofibrate. 
A

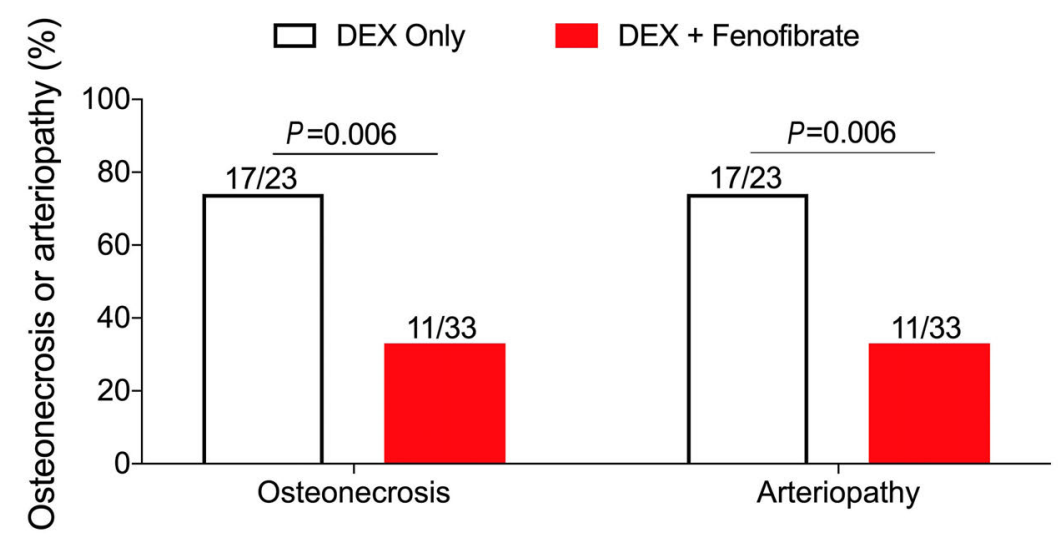

B

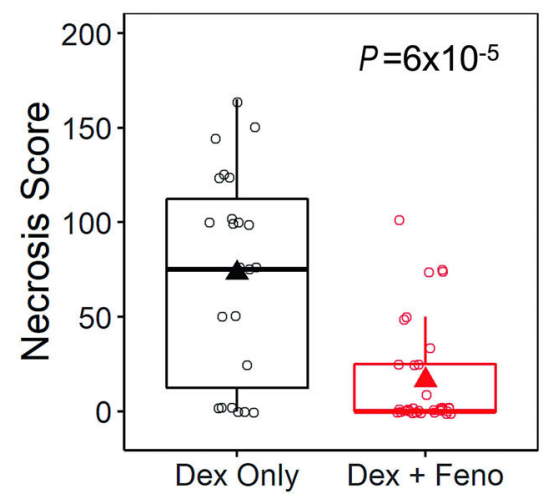

fenofibrate + dexamethasone $(P>0.99$ at each time point). By week 6 , triglycerides were higher in the mice treated with fenofibrate + dexamethasone than in those treated with fenofibrate only $(P=0.005)$ : however, levels were comparable to those in control mice $(P>0.99)$ (Figure 2A).

In dexamethasone-treated mice, fenofibrate reduced white adipose tissue: perigonadal fat (representative of visceral fat) was reduced from $0.30 \mathrm{~g}(95 \% \mathrm{CI}$ : 0.22 - 0.54) to $0.17 \mathrm{~g}(95 \% \mathrm{CI}: 0.11-0.20 ; P=0.01)$ and inguinal fat (representative of posterior subcutaneous fat) was reduced from $0.25 \mathrm{~g}(95 \% \mathrm{CI}: 0.19-0.47)$ to $0.12 \mathrm{~g}(95 \%$ CI: $0.10-0.15 ; P=0.0003$ ) (Figure $2 \mathrm{~B}$ ).

As expected, only mice that received dexamethasone developed osteonecrosis and/or arteriopathy (Figure 3). The frequency of both osteonecrosis and arteriopathy was $74 \%$ in mice treated with dexamethasone only versus $33 \%$ in those treated with dexamethasone + fenofibrate $(P=0.006)$ (Figure 3A). Not only was the frequency of osteonecrosis and arteriopathy reduced over 2 -fold with fenofibrate, but the severity of these conditions was significantly reduced. In the mice treated with dexamethasone only, the necrosis score was reduced from 75 to 0 with fenofibrate supplementation $\left(P=6 \times 10^{-5}\right)$ (Figure 3B) and the arteriopathy score was reduced from 4 to 0 $(P=0.0006)$ (Figure 3C).

In mice treated with dexamethasone + fenofibrate, there was a correlation between the triglyceride level at week 3 and osteonecrosis: mice that developed osteonecrosis had a median triglyceride concentration of $67.5 \mathrm{mg} / \mathrm{dL}$ ( $95 \%$ CI: $21.1-96.9$ ) versus $37.2 \mathrm{mg} / \mathrm{dL}$ ( $95 \% \mathrm{CI}$ : 30.9-53.1) in osteonecrosis-negative mice $(P=0.03)$ (Online Supplementary Figure S1A). By the end of treatment, there was no difference in triglyceride levels by osteonecrosis status $(P>0.99)$ (Online Supplementary Figure S1B).

At the end of treatment, there was no difference in plasma dexamethasone levels between mice treated with dexamethasone only (26.6 nM, 95\% CI: 20.0-58.3 nM) and those treated with dexamethasone + fenofibrate (20.5 nM, 95\% CI: 16.7- 25.9 nM; $P=0.1$ ) (Online Supplementary Figure S2A). The levels of fenofibric acid were significantly lower in animals treated with dexamethasone + fenofibrate $(1.9 \mu \mathrm{g} / \mathrm{mL}, 95 \%$ CI: $1.6-2.4$ $\mu \mathrm{g} / \mathrm{mL})$ than in those treated with fenofibrate only $(6.7$ $\mu \mathrm{g} / \mathrm{mL}, \quad 95 \% \quad$ CI: $6.1-8 \mu \mathrm{g} / \mathrm{mL}, \quad P<1 \times 10^{-6}$ ) (Online Supplementary Figure S2B).

\section{Fenofibrate in experimental $\mathrm{BCR}-\mathrm{ABL}^{+}$acute lymphoblastic leukemia}

In a $\mathrm{BCR}-\mathrm{ABL}^{+}$dexamethasone-sensitive model of murine ALL, (Figure 4A), all mice that did not receive dexamethasone succumbed to disease by day 35 (Figure 4C), and animals in both the groups treated with dexamethasone only and dexamethasone + fenofibrate lived longer than those that did not receive dexamethasone $\left(P<1 \times 10^{-6}\right)$. Mice were monitored for relapse until the end of follow-up at day 63. There was no difference in disease burden between the dexamethasone-only versus dexamethasone + fenofibrate treatment groups, as indicated by survival $(P=0.49)$ (Figure $4 \mathrm{C})$, spleen weight $(P>0.99)$ (Online Supplementary Figure S3A), and white blood cell count $(P>0.99)$ (Online Supplementary Figure S3B). Among the dexamethasone-treated mice, fenofibrate decreased triglyceride levels from $89.9 \mathrm{mg} / \mathrm{dL}$ to $29.0 \mathrm{mg} / \mathrm{dL}$ $(P=0.001)$ (Figure 4D). 


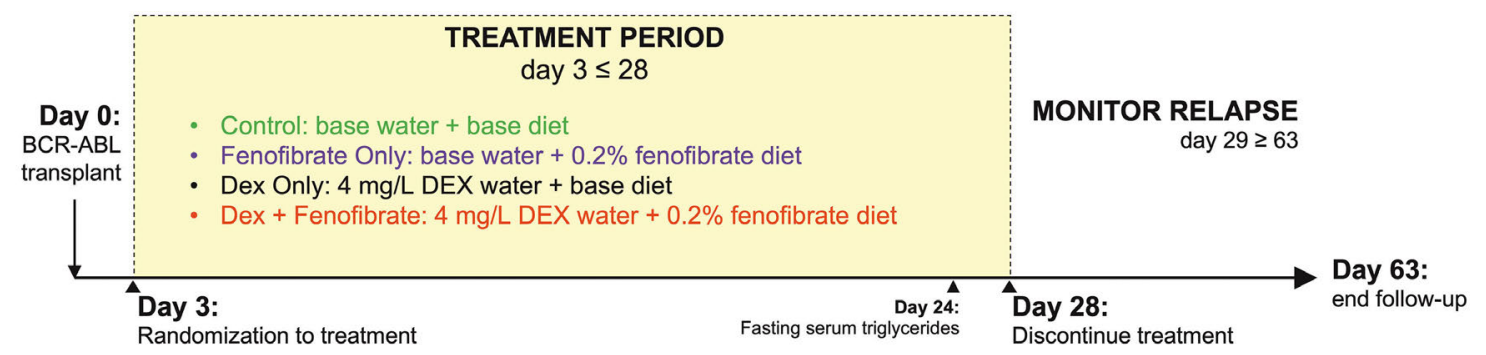

B

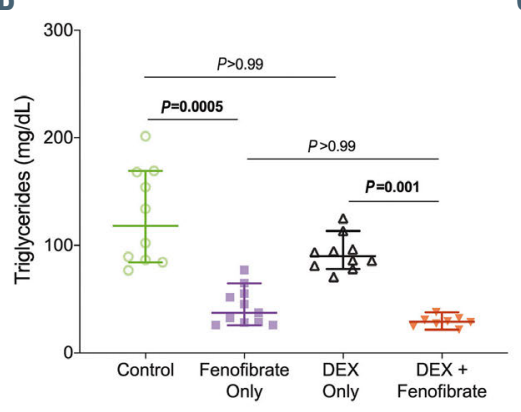

C

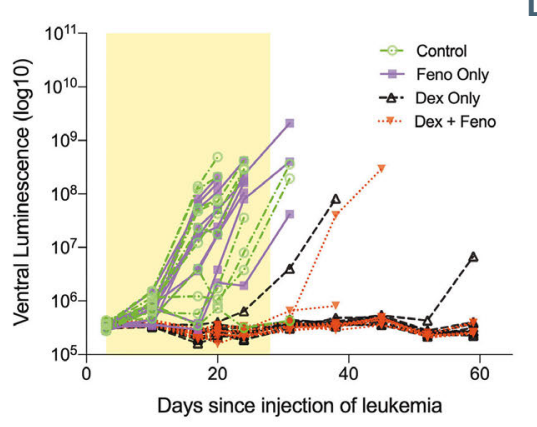

D

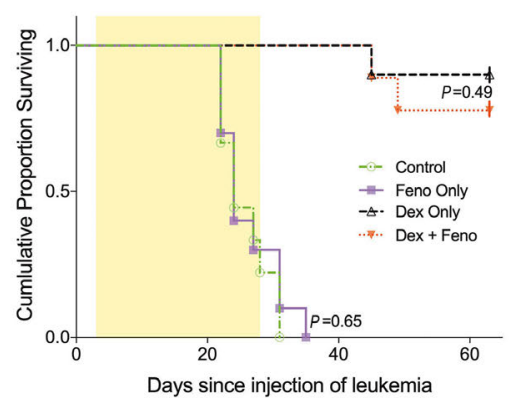

Figure 4. Fenofibrate does not affect efficacy of dexamethasone in BCR-ABL ${ }^{+}$acute lymphoblastic leukemia. (A) Experimental design. DEX water: continuous dexamethasone in drinking water; base-water: drinking water; base-diet: folic acid-deficient diet. (B) Ventral luminescence was measured weekly. (C) A log-rank test of survival data shows $P$-values for the comparisons between the various groups: control mice, mice treated with fenofibrate only, dexamethasone (DEX) only and DEX + fenofibrate. $P<1 \times 10^{6}$ between DEX-treated groups (DEX only and DEX + fenofibrate) versus no DEX treatment (control and fenofibrate only). The shaded yellow area indicates time on therapy. (D) Fasting serum triglyceride levels at day 24 (3 weeks after BCR-ABL transplant). N=9-10/group.

\section{Discussion}

Although the etiology of osteonecrosis is not completely understood, the involvement of lipid metabolism has been well documented. ${ }^{35 \cdot 37}$ However, there have been few studies investigating whether pharmacologically lowering triglycerides prevents osteonecrosis. ${ }^{38}$

We found that fenofibrate supplementation successfully managed dexamethasone-induced hypertriglyceridemia (Figure 2) and was associated with reductions in the frequency and severity of osteonecrosis (Figure 3 ). The correlation between week 3, but not week 6 triglyceride levels (Online Supplementary Figure S1), and osteonecrosis suggest that management of triglycerides is especially important early in the progression of the disease. Although there was an increase in triglyceride levels between mice treated with fenofibrate + dexamethasone and those treated with fenofibrate only by week 6 (Figure 2A), fenofibrate was able to maintain triglyceride levels comparable to those in control mice, even with prolonged continuous exposure to dexamethasone.

Any addition to ALL therapy must be thoroughly assessed to ensure that it does not interfere with the highly effective modern drug regimens. For our purposes, we utilized a model of BCR-ABL ${ }^{+}$ALL known to be sensitive to dexamethasone treatment. Fenofibrate supplementation did not cause observable differences in the efficacy of dexamethasone in the treatment of experimental BCR-ABL ${ }^{+}$ ALL (Figure 4).

In our experimental models, the dosage of both dexamethasone and fenofibrate were well tolerated (Figure 1C) and resulted in plasma levels of the drug/metabolites in the

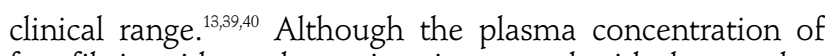
fenofibric acid was lower in mice treated with dexamethasone + fenofibrate than in those treated with fenofibrate only (Online Supplementary Figure S2B), it was still effective in reducing dexamethasone-induced hypertriglyceridemia (Figure 2A) and osteonecrosis (Figure 3), while not affecting $\mathrm{BCR}-\mathrm{ABL}^{+} \mathrm{ALL}$ treatment (Figure 4). Statins have been suggested as possible agents to reduce osteonecrosis ${ }^{41-43}$ but because they have potent interactions with CYP3A and with SLCO1B1 (enzymes involved in the metabolism and disposition of multiple ALL drugs), their likelihood of drug interactions makes them less desirable agents to use in patients with ALL.

Dexamethasone treatment is associated with hypertriglyceridemia. ${ }^{1,3,44}$ It has been shown that elevated levels of serum triglyceride-rich lipoproteins contribute to endothelial dysfunction and subsequent atherosclerosis ${ }^{45} \mathrm{We}$ hypothesized that dexamethasone-induced arteriopathy and subsequent osteonecrosis are related to hypertension caused by endothelial dysfunction. ${ }^{27,46}$ We therefore suspect that the mechanism by which fenofibrate reduced the incidence of osteonecrosis and arteriopathy was by lowering the dexamethasone-induced elevation of triglyceride-rich lipoproteins and thereby mitigating the effects of endothelial dysfunction.

In children with ALL, there are no specific guidelines for managing triglyceride levels during glucocorticoid and/or asparaginase treatment. However, conservative use of fibrates has been successful in managing triglycerides in specific cases, with no reported adverse events. ${ }^{3,5,47}$ In general, the use of fibrates in children is limited to cases of extreme hypertriglyceridemia to prevent pancreatitis, pri- 
marily in individuals with familial lipid disorders. ${ }^{20,48.50}$ Fibrates have been shown to reduce triglycerides effectively in children, by between $39 \%$ and $54 \% .49,51$ As seen in adults, data suggest that fibrates are well tolerated in children, with the most frequently reported adverse reactions being gastrointestinal disturbances and muscle cramps. ${ }^{13,14,20,49}$ However, transient increases in liver function tests have been reported in a small number of cases, ${ }^{48,51}$ indicating a potential for hepatotoxicity when used in combination with other hepatotoxic chemotherapeutic agents.

Although fibrates have been included in a case-by-case basis to manage hypertriglyceridemia during ALL thera$\mathrm{py}^{3,5,47}$ there has been no systematic analysis of the effect of lipid-lowering interventions and long-term outcomes in ALL survivors. Our proof-of-principle preclinical experiments show that fenofibrate treatment is a potential intervention to reduce dexamethasone-induced osteonecrosis in ALL, without carrying the risk of substantial drug interactions and without any untoward effect on the efficacy of ALL therapy. Our findings support the rationale for controlled clinical trials with fenofibrate in chemotherapyrelated hypertriglyceridemia.

\section{Disclosures}

No conflicts of interest to disclose.

\section{Contributions}

ERF: conceptualization of experiments; ERF, MAP,DAJ, XC, $L L, S E K, M V R$ and LJJ: methodology and data collection; $E R F$, MVR and LJJ: formal analysis; ERF, MVR and LJJ wrote the original manuscript. All authors reviewed and approved the manuscript.

\section{Acknowledgments}

We are grateful to St. Jude's Animal Resource Center and Veterinary Pathology Core for technical assistance.

\section{Funding}

This research was supported by the National Institutes of Health (CA142665, CA21765, and GM115279) and ALSAC. The content is solely the responsibility of the authors and does not necessarily represent the official views of the National Institutes of Health.

\section{References}

1. Steinherz PG. Transient, severe hyperlipidemia in patients with acute lymphoblastic leukemia treated with prednisone and asparaginase. Cancer. 1994;74(12):32343239.

2. Parsons SK, Skapek SX, Neufeld EJ, et al. Asparaginase-associated lipid abnormalities in children with acute lymphoblastic leukemia. Blood. 1997;89(6):1886-1895.

3. Bhojwani D, Darbandi R, Pei D, et al. Severe hypertriglyceridaemia during therapy for childhood acute lymphoblastic leukaemia. Eur J Cancer. 2014;50(15):2685-2694.

4. Place AE, Stevenson KE, Vrooman LM, et al. Intravenous pegylated asparaginase versus intramuscular native Escherichia coli Lasparaginase in newly diagnosed childhood acute lymphoblastic leukaemia (DFCI 05001): a randomised, open-label phase 3 trial. Lancet Oncol. 2015;16(16):1677-1690.

5. Cohen H, Bielorai B, Harats D, Toren A, Pinhas-Hamiel $O$. Conservative treatment of L-asparaginase-associated lipid abnormalities in children with acute lymphoblastic leukemia. Pediatr Blood Cancer. 2010;54(5):703-706.

6. Salvador C, Entenmann A, Salvador R, Niederwanger A, Crazzolara R, Kropshofer $\mathrm{G}$. Combination therapy of omega-3 fatty acids and acipimox for children with hypertriglyceridemia and acute lymphoblastic leukemia. J Clin Lipidol. 2018;12(5):12601266.

7. Finch ER, Smith CA, Yang W, et al. Asparaginase formulation impacts hypertriglyceridemia during therapy for acute lymphoblastic leukemia. Pediatr Blood Cancer. 2020;67(1):e28040.

8. Mogensen SS, Schmiegelow K, Grell K, et al. Hyperlipidemia is a risk factor for osteonecrosis in children and young adults with acute lymphoblastic leukemia. Haematologica. 2017;102(5):e175-e178.

9. Grundy SM, Stone NJ, Bailey AL, et al. 2018 AHA/ACC/AACVPR/AAPA/ABC/ACPM/ ADA/AGS/APhA/ASPC/NLA/PCNA guideline on the management of blood cholesterol: a report of the American College of Cardiology/American Heart Association
Task Force on Clinical Practice Guidelines. Circulation. 2019;139(25):e1082-e1143.

10. Bellosta S, Corsini A. Statin drug interactions and related adverse reactions. Expert Opin Drug Saf. 2012;11(6):933-946.

11. Wiggins BS, Saseen JJ, Page RL 2nd, et al. Recommendations for management of clinically significant drug-drug interactions with statins and select agents used in patients with cardiovascular disease: a scientific statement from the American Heart Association. Circulation. 2016;134(21):e468e495.

12. Bellosta S, Corsini A. Statin drug interactions and related adverse reactions: an update. Expert Opin Drug Saf. 2018;17(1):25-37.

13. Balfour JA, McTavish D, Heel RC. Fenofibrate. A review of its pharmacodynamic and pharmacokinetic properties and therapeutic use in dyslipidaemia. Drugs. 1990;40(2):260-290.

14. Davidson MH, Armani A, McKenney JM, Jacobson TA. Safety considerations with fibrate therapy. Am J Cardiol. 2007;99(6A): 3C-18C.

15. McKeage K, Keating GM. Fenofibrate: a review of its use in dyslipidaemia. Drugs. 2011;71(14):1917-1946.

16. Branchi A, Fiorenza AM, Rovellini A, et al. Lowering effects of four different statins on serum triglyceride level. Eur J Clin Pharmacol. 1999;55(7):499-502.

17. Besseling J, Hovingh GK, Huijgen R, Kastelein JJP, Hutten BA. Statins in familial hypercholesterolemia: consequences for coronary artery disease and all-cause mortality. J Am Coll Cardiol. 2016;68(3):252-260.

18. Staels B, Dallongeville J, Auwerx J, Schoonjans K, Leitersdorf E, Fruchart JC. Mechanism of action of fibrates on lipid and lipoprotein metabolism. Circulation. 1998;98(19):2088-2093.

19. Sirtori CR, Montanari G, Gianfranceschi G, Sirtori M, Galli G, Bosisio E. Correlation between plasma levels of fenofibrate and lipoprotein changes in hyperlipidaemic patients. Eur J Clin Pharmacol. 1985;28(6): 619-624.

20. Kennedy MJ, Jellerson KD, Snow MZ, Zacchetti ML. Challenges in the pharmacologic management of obesity and secondary dyslipidemia in children and adolescents. Paediatr Drugs. 2013;15(5):335-342.

21. Yang L, Boyd K, Kaste SC, Kamdem Kamdem L, Rahija RJ, Relling MV. A mouse model for glucocorticoid-induced osteonecrosis: effect of a steroid holiday. J Orthop Res. 2009;27(2):169-175.

22. Kawedia JD, Janke L, Funk AJ, et al. Substrain-specific differences in survival and osteonecrosis incidence in a mouse model. Comp Med. 2012;62(6):466-471.

23. Ramsey LB, Janke LJ, Payton MA, et al. Antileukemic efficacy of continuous vs discontinuous dexamethasone in murine models of acute lymphoblastic leukemia. PLoS One. 2015;10(8):e0135134.

24. Murai T, Yamada T, Miida T, Arai K, Endo N, Hanyu T. Fenofibrate inhibits reactive amyloidosis in mice. Arthritis Rheum. 2002;46(6):1683-1688

25. Oosterveer MH, Grefhorst A, van Dijk TH, et al. Fenofibrate simultaneously induces hepatic fatty acid oxidation, synthesis, and elongation in mice. J Biol Chem 2009;284(49):34036-34044.

26. Huang J, Das SK, Jha P, et al. The PPARalpha agonist fenofibrate suppresses B-cell lymphoma in mice by modulating lipid metabolism. Biochim Biophys Acta. 2013;1831(10): 1555-1565.

27. Janke LJ, Liu C, Vogel P, et al. Primary epiphyseal arteriopathy in a mouse model of steroid-induced osteonecrosis. Am J Pathol. 2013;183(1):19-25.

28. Liu C, Janke LJ, Kawedia JD, et al. Asparaginase potentiates glucocorticoidinduced osteonecrosis in a mouse model. PLoS One. 2016;11(3):e0151433.

29. Finch ER, Janke LJ, Smith CA, et al. Bloodstream infections exacerbate incidence and severity of symptomatic glucocorticoidinduced osteonecrosis. Pediatr Blood Cancer. 2019;66(6):e27669.

30. Williams RT, Roussel MF, Sherr CJ. Arf gene loss enhances oncogenicity and limits imatinib response in mouse models of Bcr-Ablinduced acute lymphoblastic leukemia. Proc Natl Acad Sci U S A. 2006;103(17):66886693.

31. Boulos N, Mulder HL, Calabrese CR, et al. Chemotherapeutic agents circumvent emer- 
gence of dasatinib-resistant BCR-ABL kinase mutations in a precise mouse model of Philadelphia chromosome-positive acute lymphoblastic leukemia. Blood. 2011;117 (13):3585-3595.

32. Ramsey LB, Janke LJ, Edick MJ, et al. Host thiopurine methyltransferase status affects mercaptopurine antileukemic effectiveness in a murine model. Pharmacogenet Genomics. 2014;24(5):263-271.

33. Team RDC. R: A Language and Environment for Statistical Computing. R Foundation for Statistical Computing, Vienna, Austria. 2006.

34. Prism. GraphPad Software, version 8.2.1. La Jolla, CA, USA.

35. Kuroda T, Tanabe N, Wakamatsu A, et al. High triglyceride is a risk factor for silent osteonecrosis of the femoral head in systemic lupus erythematosus. Clin Rheumatol. 2015;34(12):2071-2077.

36. Mankin HJ. Nontraumatic necrosis of bone (osteonecrosis). N Engl J Med. 1992;326(22): 1473-1479.

37. Sakamoto Y, Yamamoto T, Sugano N, et al. Genome-wide association study of idiopathic osteonecrosis of the femoral head. Sci Rep. 2017;7(1):15035.

38. Lee YJ, Cui Q, Koo KH. Is there a role of pharmacological treatments in the prevention or treatment of osteonecrosis of the femoral head? A systematic review. J Bone
Metab. 2019;26(1):13-18.

39. Ito C, Evans WE, McNinch L, et al. Comparative cytotoxicity of dexamethasone and prednisolone in childhood acute lymphoblastic leukemia. J Clin Oncol. 1996;14(8):2370-2376.

40. Kawedia JD, Liu C, Pei D, et al. Dexamethasone exposure and asparaginase antibodies affect relapse risk in acute lymphoblastic leukemia. Blood. 2012;119(7): 1658-1664.

41. Pritchett JW. Statin therapy decreases the risk of osteonecrosis in patients receiving steroids. Clin Orthop Relat Res. 2001;(386): 173-178.

42. Pengde K, Fuxing P, Bin S, Jing Y, Jingqiu C. Lovastatin inhibits adipogenesis and prevents osteonecrosis in steroid-treated rabbits. Joint Bone Spine. 2008;75(6):696-701.

43. Ajmal M, Matas AJ, Kuskowski M, Cheng EY. Does statin usage reduce the risk of corticosteroid-related osteonecrosis in renal transplant population? Orthop Clin North Am. 2009;40(2):235-239.

44. Peckett AJ, Wright DC, Riddell MC. The effects of glucocorticoids on adipose tissue lipid metabolism. Metabolism. 2011;60(11): 1500-1510.

45. Peng J, Luo F, Ruan G, Peng R, Li X. Hypertriglyceridemia and atherosclerosis. Lipids Health Dis. 2017;16(1):233.
46. Janke LJ, Van Driest SL, Portera MV, et al. Hypertension is a modifiable risk factor for osteonecrosis in acute lymphoblastic leukemia. Blood. 2019;134(12):983-986.

47. Therrien R, Barret P, Robitaille M, Moghrabi A. Use of fenofibrate in asparaginaseinduced hypertriglyceridemia in children with ALL: a case series. http://wwwindicibleca/urpp/20130516_FENOFIBRATE_Anna lespdf. 2013

48. Wheeler KA, West RJ, Lloyd JK, Barley J. Double blind trial of bezafibrate in familial hypercholesterolaemia. Arch Dis Child. 1985;60(1):34-37.

49. Smalley CM, Goldberg SJ. A pilot study in the efficacy and safety of gemfibrozil in a pediatric population. J Clin Lipidol. 2008;2(2):106-111.

50. Expert Panel on Integrated Guidelines for Cardiovascular Health and Risk Reduction in Children and Adolescents: National Heart, Lung and Blood Institute. Expert panel on integrated guidelines for cardiovascular health and risk reduction in children and adolescents: summary report. Pediatrics. 2011;128(Suppl 5):S213-56.

51. Steinmetz J, Morin C, Panek E, Siest G, Drouin P. Biological variations in hyperlipidemic children and adolescents treated with fenofibrate. Cli Chim Acta. 1981;112(1):4353. 